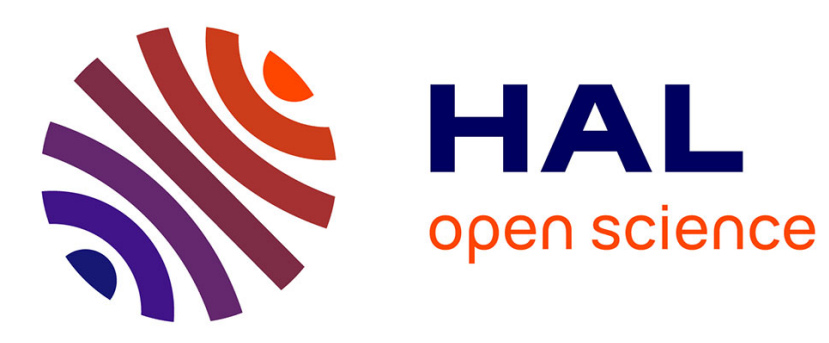

\title{
JeB: Safe Simulation of Event-B Models in JavaScript
}

Faqing Yang, Jean-Pierre Jacquot, Jeanine Souquières

\section{To cite this version:}

Faqing Yang, Jean-Pierre Jacquot, Jeanine Souquières. JeB: Safe Simulation of Event-B Models in JavaScript. The 20th Asia-Pacific Software Engineering Conference (APSEC), Dec 2013, Bangkok, Thailand. hal-00908056

\section{HAL Id: hal-00908056 https://hal.inria.fr/hal-00908056}

Submitted on 7 Dec 2013

HAL is a multi-disciplinary open access archive for the deposit and dissemination of scientific research documents, whether they are published or not. The documents may come from teaching and research institutions in France or abroad, or from public or private research centers.
L'archive ouverte pluridisciplinaire HAL, est destinée au dépôt et à la diffusion de documents scientifiques de niveau recherche, publiés ou non, émanant des établissements d'enseignement et de recherche français ou étrangers, des laboratoires publics ou privés. 


\title{
JeB: Safe Simulation of Event-B Models in JavaScript
}

\author{
Faqing Yang, Jean-Pierre Jacquot and Jeanine Souquières \\ Université de Lorraine - LORIA (UMR 7503) \\ F-54506 Vandœuvre lès Nancy, France \\ Email: \{firstname.lastname\}@loria.fr
}

\begin{abstract}
The validation of formal models is a challenge for formal methods. We propose JeB, a framework which generates and executes simulations of Event-B models, even highly nondeterministic ones. JeB allows users to safely insert pieces of code to supply deterministic computations where the automatic translation fails. We present how JeB translates Event-B model into JavaScript. We define Fidelity as the formal notion which captures the idea of the correctness of a simulation. We define it through proof-obligations.
\end{abstract}

Keywords-Formal methods, Event-B, Validation, Simulation, JavaScript, Proof-Obligations

\section{INTRODUCTION}

Formal methods promise the production of software which are correct, i.e., proven to meet their specification. Hence, getting the right formal specification is crucial.

The validation of formal models through execution is an old issue [1], [2], [3]. Actually, the main difficulty revolves around the level of determinism: good practice advocates the slow refinement of highly abstract and non-deterministic specifications [4] while execution tools require deterministic or quasi-deterministic models.

Event-B [5] is a modeling language of the B family [11] based on refinement; it is supported by the Rodin environment [6]. Event-B has a dynamic semantics which is implemented by the available compilers [7], [8] and animators [9], [10]. However, while the semantics accounts well for nondeterminism, the current tools often fail on early models in developments. Hence, those cannot be validated.

JeB, a JavaScript framework for executing Event-B specifications, answers this issue. JeB consists of a translator which compiles the Event-B model into JavaScript code and generates enough "hooks" for developers to provide their own hand-coded resolution of non-determinism. Obviously, hand-coded functions could be "incorrect." Fidelity formally expresses this notion of correctness. It is defined so we can derive proof obligations.

JavaScript looks an unlikely choice in a formal context, yet it has technical features which fit well with our purpose. Furthermore, the browsers running JavaScript allow to set up easily graphical displays.

We first present Event-B. Then, we discuss JeB's design and the translator's implementation. Last, we show the formal definition of fidelity and the associated proof obligations.

\section{EVENT-B}

\section{A. Notations}

A specification in Event-B consists of a state and events. The state maps names to values; it is constrained by an invariant. For practical purposes, models are split into Contexts and Machines. The values are built inductively from integers, booleans and symbols by using power sets and cartesian products. Special cases such as binary relations, partial functions, or injections enjoy specific notations. The typing system is equivalent to set membership and inclusion. The invariant is a first-order formula on the state. It is a conjunction of smaller formulas called axioms in contexts and invariants in machines.

Events model the evolution of the state. Formally, they are guarded substitutions. Guards are first-order formulas on the state; they may contain free variables called event parameters. Substitutions change some values, all at the same time. An event is enabled when its guard is true, it can then be fired.

The formal semantics of an Event-B model is based on the feasibility ( fis) property expressing two ideas:

- a legal state with actual values must exist, and

- firing enabled events leads from legal states to legal states. The specification language is designed so that fis can be cast as a set of small logical formulas, called proof-obligations. A model is correct when all its POs have been discharged.

Figure 1 shows the first model of a platooning system. Vehicles are required to maintain a minimum distance $d m i n$ between themselves. At this level, the distance function dist, the representation of the geometric surface Plane and the movement of the platoon move are abstractions.

\section{B. Refinement}

Event-B embodies two ideas about programs: they are elaborated by stepwise refinements, and they can be correct by construction. In this context, correct means "mathematically proven to meet their specification."

A model $M_{r}$ is a refinement of a model $M_{a}$, if:

- $M_{r}$ is consistent (i.e., it defines one actual state, invariants are preserved through the actions),

- there is an abstraction function from the $M_{r}$ state and events to the $M_{a}$ state and events,

- $M_{r}$ legal states are abstracted as $M_{a}$ legal states, and

- all events fired in $M_{r}$ preserve the invariant of $M_{a}$. 
Table I

NAMESPACES USED IN THE SIMULATOR CODE (EXCERPT)

\begin{tabular}{lll}
\hline Namespace & Prefix & Description \\
\hline jeb & jeb & the top namespace \\
jeb.axiom & \$axm & axioms \\
jeb.event & \$evt & machine events \\
\$evt.<eventId $>$ arg & \$arg & event parameters \\
\$B & \$B & JavaScript library for Event-B \\
\hline
\end{tabular}

Table II

STRUCTURAL MAPPING OF A CONTEXT

\begin{tabular}{ll}
\hline Event-B & JavaScript/HTML \\
\hline CONTEXT name & $\begin{array}{l}\text { (1) name.js (context model) } \\
\text { (2) name.html (user interface) }\end{array}$ \\
EXTENDS context_names & $\begin{array}{l}\text { list of context_name in the HTML page } \\
\text { for navigation }\end{array}$ \\
SETS identifiers & $\begin{array}{l}\text { list of \$cst.identifier } \\
\text { CONSTANTS identifiers }\end{array}$ \\
$\begin{array}{l}\text { list of \$cst.identifier } \\
\text { AXIOMS predicates }\end{array}$ & list of jeb.lang.Axiom instances \\
END &
\end{tabular}

\section{JEB REALIZATION}

Technically, a simulation consists in three parts: the generated code, a runtime library, and the hand-coded functions provided by the users.

\section{A. Generated code}

1) Namespace: Generated names must not conflict between themselves or existing names in the target environment, and should be traceable back to Event-B. With prefixes on global object properties, we can achieve the same effect as with namespaces; Table I shows some examples.

2) Translation of Contexts: Each context is translated into a file which contains the translation of the axioms as JavaScript objects. The overall structure of the file is derived from the structure of the Event-B code, as shown on Table II.

a) Sets and Constants: They are translated as standard identifiers prefixed by \$cst. They are given values in the configuration files. Enumerated sets are instantiated by collecting elements already defined in the CONSTANTS clause. The JeB library supports the instantiation of a carrier set either as a collection of symbolic strings or as a class in the OOP sense.

b) Axioms: JeB translates each axiom as an instance of jeb.lang. Axiom, prefixed by \$axm:

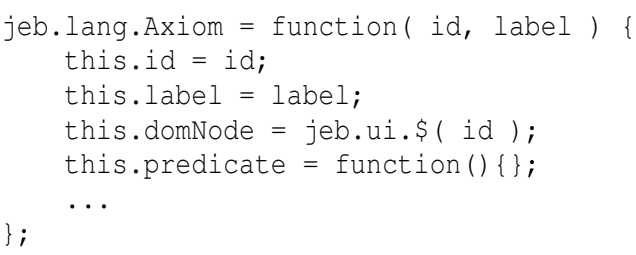

where $i d$ is a unique reference; label is axiom's name as written in the context; domNode links this instance to the HTML page to display its evaluation; predicate is a method to evaluate the axiom.
3) Translation of Machines: The translation of a machine generates three files: an executable version of the model, a configuration file (both JavaScript), and a simulation GUI (HTML). The configuration file contains stubs and default implementation of non-deterministic features.

a) Variables: Each variable is translated as an instance of jeb.lang.Variable, prefixed by \$var:

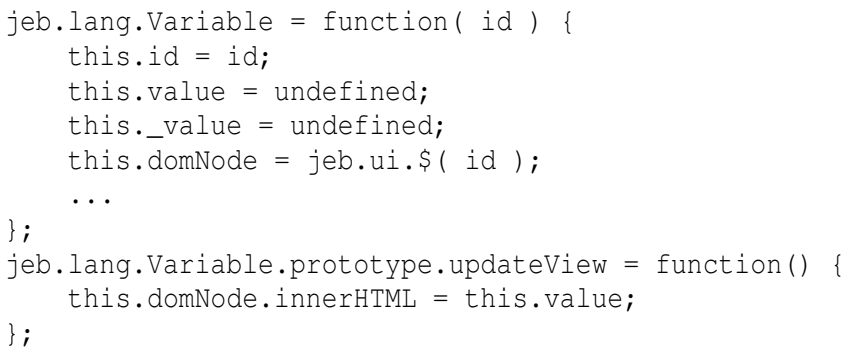

where $i d$ is a unique reference; value is the current value; _value is the primed value; domNode binds to an element in the HTML page which displays its value; updateview is the method used for updating the user interface. The variable value can be any Event-B mathematical object. It is checked by computing the invariant after each simulation cycle.

b) Invariants: They are translated like axioms, using jeb.lang. Invariant instead of jeb.lang. Axiom.

c) Events: JeB generates an independent simulation for each refinement, so the refinement links (refines and with) are ignored. The guards and actions are translated as two different functions. Events' parameters require a special treatment described thereafter. Each event is translated as an instance of jeb. lang. Event:

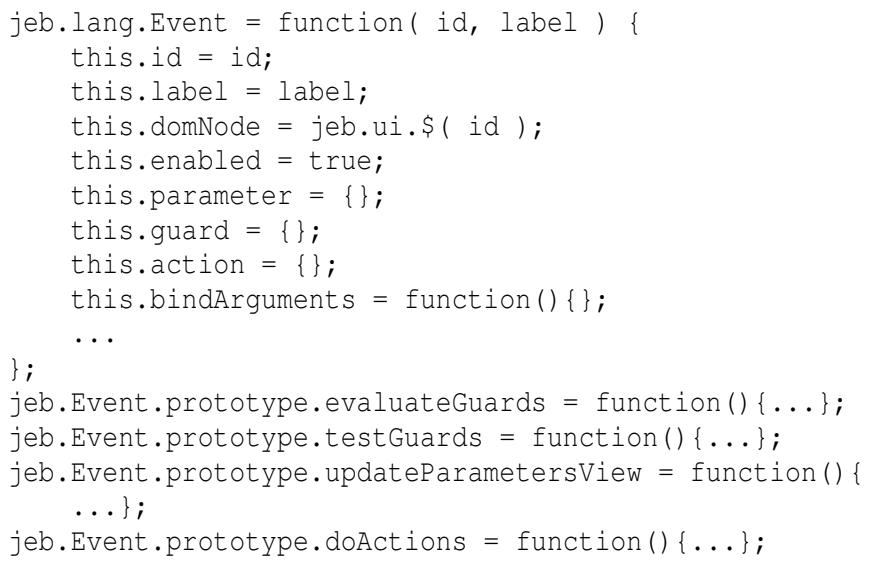

where id, label, and domNode have the same role as for axioms. The property enabled contains the result of the evaluation of the guards. The properties parameter, guard, and action are namespaces for the event's parameters, guards and actions, respectively. The four functions are helpers for implementing the simulation cycle: evaluateGuards and testGuards are used for determining parameter values, updateParametersView and doActions do what their names imply.

d) Event Parameters: The so-called "event parameters" in Event-B are the variables introduced in the ANY clause 


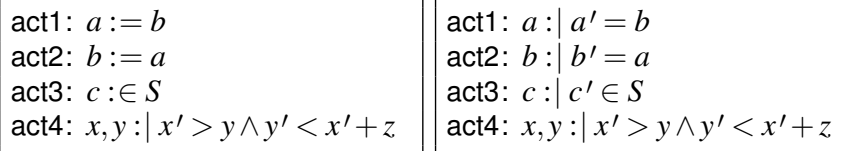

act1: $a: \mid a^{\prime}=b$

act2: $b: \mid b^{\prime}=a$

act3: $c: \mid c^{\prime} \in S$

act4: $x, y: \mid x^{\prime}>y \wedge y^{\prime}<x^{\prime}+z$

Figure 2. Assignments

of events. They are of two kinds: deterministic variables are actually local variables, their value is automatically computed by JeB; non deterministic variables are true parameters in the common programming sense.

Each true parameter par is associated with an argument generator function get_par which is bound to the input field in the HTML page. The JeB translator generates it into a configuration file as a function stub or, when possible, as a default implementation. Users should provide their own implementation for a realistic simulation in the auto-run mode of execution. Before each simulation cycle, the bindArguments method binds each parameters to a get_par function, which can then be changed "on the fly."

The order in which the parameters are declared is irrelevant in Event-B, but not in JavaScript. When necessary, the JeB translator adjusts the order of parameters in the GUI and in the bindArguments function so they get a value before being used.

Each identifier declared in an ANY clause is translated as an instance of jeb.lang.Parameter:

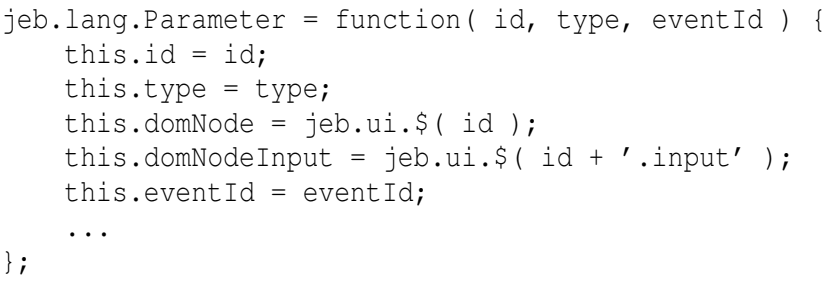

where $i d$ is a unique reference; type indicates its nature (local variable or true parameter); domNode (resp. domNodeInput) binds to an element in the HTML page for display (resp. input), event $I d$ references the event;

e) Event Guards: An event guard is a predicate formula. The enabled status of an event is the result of computing all its guards. Guards are translated like axioms with an added reference to the event.

f) Event Actions: Actions are assignments with two features: they occur simultaneously and they can involve nondeterministic values ("becomes such that" for instance).

The left-hand side of Figure 2 is a classical trap. The idea is to use only :| assignments and "primed values" as shown on the right-hand side. The order of execution becomes then irrelevant. Technically, the assignments are realized on the variables' property _value and copied to the value property after all assignments have been made.

The non-deterministic assignments generate stubs in the configuration file which call default implementation in the runtime library. Users may override them to provide their own, deterministic, implementation.
Each action is coded as an instance of

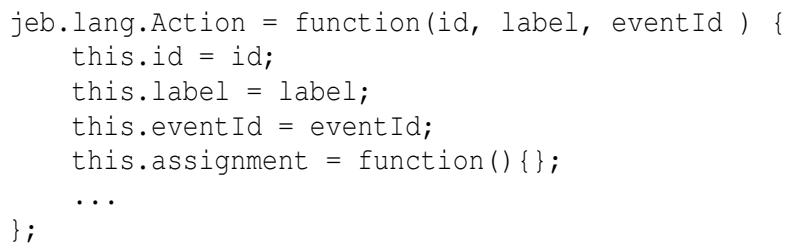

where assignment is the method to realize the assignment.

4) Translation of Formulas: Each operator is coded as a call to the runtime library. The API of the library is a oneto-one mapping of the Event-B mathematical notation. So, the translation algorithm is based on Event-B syntax and independent of the target language. Proving the correctness of the translation resolves into proving the library functions. Table III shows some translation rules.

\section{B. Runtime Library}

1) Simulation Scheduler: The operational semantics is implemented as follows:

(1) setup contexts, prompt user if needed

(2) trigger the INITIALISATION event

(3) do

(1) save the state of variables

(2) update the variable view

(3) compute the invariants, stop if one is false

(4) build the set of enabled events, stop if empty

(5) pick an enabled event to trigger

(6) save the state of arguments

(7) execute the actions of the selected event

The step (3.3) is useless when executing a fully proven model, but it is a useful feature for the preliminary exploration of complex refinements. Step (3.4) is a computation of all the guards of the events based on the state values and the values chosen for the parameters, either through user's input in the GUI or computation of the get_par functions.

2) JavaScript Library for Event-B: The computation core of the expressions is delegated to a library which implements set theory and first order logic. Its realization aims at two goals: a clear interface and efficient computations.

Technically, the APIs are modeled on the mathematical language of Event-B. The translation from Event-B to JavaScript is mostly syntactic. The APIs contain about 90 profiles.

The representation of numbers is an important issue. In Event-B, integers are unbounded; contrary to JavaScript primitive numbers. Discretization of computations, tiny units or large multiplicative constants are standard modeling tricks to model $\mathbb{R}$ or functions such as sin. They induce very large numbers. The library implements arbitrary large integers.

3) Graphical User Interface: The simulation interface is implemented as an HTML page [14]. JeB provides a generic page with the code needed to control it.

\section{Hand-Coded Add-Ons}

An important feature of $\mathrm{JeB}$ is to allow users to provide safely pieces of code to improve the simulation. 
Table III

FORMULA TRANSLATION RULES (EXCERPTS) ( $T R$ AND ${ }^{T R *}$ DENOTES RECURSIVE CHILD TREe TRAVERSAL)

\begin{tabular}{|c|c|c|}
\hline Event-B Notation & Event-B Syntax & Translation \\
\hline & Predicates & \\
\hline True predicate & $\top$ & $\$ B . b T r u e()$ \\
\hline Implication & $P \Rightarrow Q$ & $\$$ B.implication $\left(P^{T R}, Q^{T R}\right)$ \\
\hline Universal & $\forall x_{1}, \ldots, x_{n} \cdot P(n \geq 1)$ & $\$ B . \operatorname{forAll}\left(P^{T R *},\left[x_{1}^{T R *}, \ldots, x_{n}^{T R *}\right]\right)$ \\
\hline \multirow[t]{2}{*}{ Set membership } & $E \in S$ & \$B.belong $\left(E^{T R}, S^{T R}\right)$ \\
\hline & identifiers / Atomic values / Expressions & \\
\hline Free identifier & $\chi$ & 1) $\$ c s t \cdot \chi$ if $\chi$ is a constant \\
\hline & & 2) \$var. $\chi$.value if $\chi$ is a variable \\
\hline & & 3) \$arg. $\chi$.value if $\chi$ is a parameter \\
\hline Boolean TRUE & TRUE & $\$ B . T R U E$ \\
\hline Bool expression & $\operatorname{bool}(P)$ & $\$ B . \operatorname{bool}\left(P^{T R}\right)$ \\
\hline Integer literal & $\alpha$ & $\$ B\left({ }^{\prime} \alpha^{\prime}\right)$ \\
\hline Bool expression & $\operatorname{bool}(P)$ & $\$ B . \operatorname{bool}\left(P^{T R}\right)$ \\
\hline \multirow[t]{2}{*}{ Subtraction } & $m-n$ & $\$ B . \operatorname{minus}\left(m^{T R}, n^{T R}\right)$ \\
\hline & Sets / Relations & \\
\hline Empty set & $\varnothing$ & \$B.EmptySet \\
\hline Interval & $m \ldots n$ & $\$ B . U p T o\left(m^{T R}, n^{T R}\right)$ \\
\hline Natural numbers & & \$B.NATURAL \\
\hline Set extension & $\left\{E_{1}, \ldots, E_{n}\right\}(n \geq 1)$ & $\$$ B.SetExtension $\left(E_{1}^{T R}, \ldots, E_{n}^{T R}\right)$ \\
\hline Ordered pair & $E \mapsto F$ & $\$$ B.Pair $\left(E^{T R}, F^{T R}\right)$ \\
\hline Relations & $S \leftrightarrow T$ & \$B.Relations $\left(S^{T R}, T^{T R}\right)$ \\
\hline Partial functions & $S \rightarrow T$ & \$B.PartialFunctions $\left(S^{T R}, T^{T R}\right)$ \\
\hline \multirow[t]{2}{*}{ Function image } & $f(E)$ & $\$ B$. functionImage $\left(f^{T R}, E^{T R}\right)$ \\
\hline & Assignments & \\
\hline Becomes such that & $x_{1}, \ldots, x_{n}: \mid Q\left(x_{1}^{\prime}, \ldots, x_{n}^{\prime}\right)(n \geq 1)$ & \$B.becomesSuchThat $\left(\left[x_{1}^{T R}, \ldots, x_{n}^{T R}\right], Q^{T R *},\left[x_{1}{ }^{\prime T R *}, \ldots, x_{n}{ }^{\prime T R *}\right]\right)$ \\
\hline
\end{tabular}

1) Graphical Display: The generic GUI contains two stubs:

- jeb.animator.init to initialize the display area,

- jeb.animator.draw to draw the system's state.

The second function is called at each cycle, the first one is called only once during initialization.

2) Parameters of Events: They are the main controls when running a simulation. The value of each "true parameter" is obtained by getting the content of the input fields in the GUI. Those fields can contain either a literal value or a function call. In the latter case, the execution mechanism looks for an implementation in the configuration files. Users can switch between values and functions at any time during a simulation.

\section{Correctness of Simulation}

The notion of correctness is captured by the idea of fidelity.

\section{A. Definition of Fidelity}

The execution of an Event-B model $M$ is formalized as a trace [15]. Intuitively, a trace is a succession of instances of firings of events ${ }^{1}$, starting with the $E^{0}$ (INITIALISATION), which goes through a succession of legal states. Formally, $E^{0} e_{1} e_{2} \ldots e_{n}$ is a trace iff

$$
\forall j . j \in 1 . . n \Rightarrow e_{j} \in \operatorname{Evts}(M)
$$

and

$$
\text { fis }\left(E^{0} ; e_{1} ; e_{2} ; \ldots e_{n}\right) \Leftrightarrow \text { true }
$$

where fis is the feasibility predicate defined as the point-wise application of the feasibility PO of the machine $M$ [11]:

$$
\operatorname{Axm}(s, c) \Rightarrow \exists v^{\prime} \cdot B A P_{E^{0}}\left(s, c, v^{\prime}\right)
$$

\footnotetext{
${ }^{1}$ lowercase $e_{i}$ denotes instances, uppercase $E^{i}$ denotes events
}

$$
\begin{gathered}
\bigwedge_{i=1}^{m} \operatorname{Axm}(s, c) \wedge \operatorname{Inv}(s, c, v) \wedge \operatorname{Grd}_{E^{i}}\left(x_{E^{i}}, s, c, v\right) \Rightarrow \\
\exists v^{\prime} \cdot \operatorname{BAP}_{E^{i}}\left(x_{E^{i}}, s, c, v, v^{\prime}\right)
\end{gathered}
$$

where $s, c, v$ are the Sets, Constants and Variables of the state, Inv and Axm the invariants and axioms, BAP the BeforeAfter predicate of a substitution, and $v^{\prime}$ is the After-value. $\operatorname{Traces}(M)$ is the set of all traces of the machine $M$.

Intuitively, fidelity is seen as the inclusion of the set of traces of the executable model into Traces $(M)$. However, one set belongs to the JavaScript world, while the other belongs to the Event-B world. So, we introduce three abstraction-functions:

- $f \in V_{J} \rightarrow V_{B}$ which maps values in JavaScript to their equivalent in Event-B,

- eval which evaluates the JavaScript translation $f_{\Psi}()$ of the Event-B predicate $\Psi$. eval is assumed such that:

$$
\begin{gathered}
\operatorname{eval}\left(x^{t}=x_{0}\right) ; \operatorname{eval}\left(f_{\Psi}()\right)==\text { true }(\text { resp. false }) \Leftrightarrow \\
\operatorname{bool}\left(\left[x:=f\left(x_{0}\right)\right] \Psi\right)=\text { TRUE }(\text { resp. FALSE })
\end{gathered}
$$

where $[a:=b] \Psi$ is the substitution of $a$ by $b$ in $\Psi$.

- $f_{\text {Trace }} \in f_{E v t s(M)} \rightarrow E v t s(M)$ which maps the JavaScript translation of each event to each Event-B event.

Assuming $f$ and $e v a l$ and noting $x_{f_{e_{j}}}$ the event's parameters, the set of "interesting" traces in a simulation is defined as

$$
f_{E^{0}}() ; f_{e_{1}}\left(x_{f_{e_{1}}}\right) ; f_{e_{2}}\left(x_{f_{e_{2}}}\right) ; \ldots ; f_{e_{n}}\left(x_{f_{e_{n}}}\right)
$$

belongs to TraceSimulation $(M)$ if

$$
\bigwedge_{j=1}^{n} \operatorname{eval}\left(f_{e_{j}} \cdot f_{\text {Grd }}\left(x_{f_{e_{j}}}\right)\right)==\text { true }
$$

The definition of fidelity is simply that all traces in TraceSimulation $(M)$ can be abstracted to a trace of $M$

$\forall t \cdot t \in \operatorname{TraceSimulation}(M) \Rightarrow \operatorname{map}\left(t, f_{\text {Trace }}\right) \in \operatorname{Traces}(M)$ 


\section{B. Proof Obligations}

The general definition can be broken down into local properties, which allow to associate a specific PO to each hand-coded element [16]. Six cases have been identified

1) Valuation of constants. The PO checks the axioms hold.

$$
\operatorname{bool}\left(\left[s, c:=f\left(s_{J}\right), f\left(c_{J}\right)\right] A x m(s, c)\right)=T R U E
$$

2) Valuation of parameters. The PO checks the guards hold.

$$
\bigwedge_{j=1}^{n} \operatorname{bool}\left(\left[x_{e_{j}}:=f\left(x_{f_{e_{j}}}\right)\right] \operatorname{Grd}_{e_{j}}\left(x_{e_{j}}, s, c, v\right)\right)=T R U E
$$

3) Parameter generator. The produced value is checked against the event's guards, no proof is needed.

4) Predicate in an invariant or a guard. Let $f_{\Psi}$ be the user implementation of the predicate $\Psi$, the PO is

$$
\operatorname{eval}\left(f_{\Psi}()\right)==\text { true } \Leftrightarrow \operatorname{bool}(\Psi)=\text { TRUE }
$$

5) Value for an action. Let $f_{a c t}$ the user implementation. The PO ensures that computed values are admissible values.

$$
\left\{f\left(v_{J}\right) \mid v_{J}=f_{a c t}()\right\} \subseteq\left\{v^{\prime} \mid B A P_{a c t}\left(x_{E^{i}}, s, c, v, v^{\prime}\right)\right\}
$$

6) Function defined by non-constructive properties. Technically, this is the most difficult case. An axiom defining a function $g$ has the form

$$
\forall v_{1}, \ldots, v_{n} \cdot \operatorname{type}\left(v_{1}\right) \wedge \ldots \wedge \operatorname{type}\left(v_{n}\right) \Rightarrow \Psi\left(g, v_{1}, \ldots, v_{n}\right)
$$

where $\Psi$ contains several application of $g$, and type $\left(v_{k}\right)$ is a typing predicate for $v_{k} . \Psi$ is translated into a program, Prog, using JeB rules for predicates and calls to the user's implementation, $g_{\text {user }}$, for each appearance of $g$. Let $f_{\Psi}$ be the translation in JavaScript of $\Psi$ using the definition of $g$, then the PO is

$$
w p\left(\operatorname{Prog}, f_{\Psi}\right) \Rightarrow \bigwedge_{k=1}^{n} f_{\text {type }}\left(v_{k}\right)
$$

where $w p$ is the usual weakest-precondition transformer.

POs 1, 2 and 5 can be discharged within the Event-B system; POs 4 and 6 must be discharged within JavaScript semantics.

\section{Assumptions}

The discussion of fidelity focused on our main contribution: the safe inclusion of hand-coded pieces of code. Discharging the POs proves the fidelity under four assumptions:

A1 Event-B values can be represented in JavaScript,

A2 Event-B expressions can be translated into an observational equivalent JavaScript expression,

A3 Event-B AST can be syntactically mapped to library calls,

A4 Event-B operational semantic cycle can be implemented into a scheduler in JavaScript.

SETL [17] shows that A1 is reasonable. Existing translators such as B2C [7] and EB2ALL [8] show that A2 makes sense. The design of our run library, whose API covers all EventB notations, establishes the validity of A3. A4 grounds tools such as ProB [9] and Brama [10]. We are confident that the assumptions can be proven. Such proofs would use the wellestablished techniques of proving compilers.

\section{CONCLUSION}

In [18] we showed how a formally correct model can be safely transformed into an "incorrect" animatable model which keeps the same behaviors. Here, we showed how to produce simulations which are guaranteed to preserve a subset of the behaviors. So, we have the theoretical framework necessary to validate most refinements in a development.

The design and implementations of support tools for proving fidelity is the next challenge: the mix of different formal frameworks raises interesting questions. Another challenge lies in the invention of techniques to create, manage and replay validation scenarios using simulations and animations.

\section{REFERENCES}

[1] R. M. Balzer, N. M. Goldman, and D. S. Wile, "Operational Specification as the Basis for Rapid Prototyping," SIGSOFT Softw. Eng. Notes, vol. 7, no. 5, pp. 3-16, 1982.

[2] N. E. Fuchs, "Specifications are (preferably) executable," Software Engineering Journal, vol. 7, pp. 323-334, September 1992.

[3] I. Hayes and C. Jones, "Specifications are not (necessarily) executable," Software Engineering Journal, vol. 4, pp. 330-338, November 1989.

[4] J.-R. Abrial, "Formal methods in industry: achievements, problems, future," in Proceedings of the 28th international conference on Software engineering, ser. ICSE '06. New York, USA: ACM, 2006, pp. 761-768.

[5] 1 , Modeling in Event-B: System and Software Engineering. Cambridge University Press, 2010.

[6] RODIN, "Rigorous Open Development Environment for Complex Systems," Website, consulted November 2012, http://www.event-b.org.

[7] S. Wright, "Automatic Generation of C from Event-B," in IM_FMT, Workshop on Integration of Model-based Formal Methods and Tools, Dusseldorf, Germany, 2009.

[8] D. Méry and N. Singh, "Automatic Code Generation from EventB Models," in Proc. Symposium on Information and Communication Technology. Hanoi, Vietnam: ACM, 2011.

[9] M. Leuschel, J. Falampin, F. Fritz, and D. Plagge, "Automated property verification for large scale B models with ProB," Formal Aspects of Computing, pp. 1-27, 2011.

[10] T. Servat, "BRAMA: A New Graphic Animation Tool for B Models," in B 2007: Formal Specification and Development in B. Springer-Verlag, 2007, pp. 274-276.

[11] J.-R. Abrial, The B Book. Cambridge University Press, 1996.

[12] T. Nakatani, T. Tsumaki, M. Tsuda, M. Inoki, S. Hori, and K. Katamine, "Requirements Maturation Analysis by Accessibility and Stability," in Software Engineering Conference (APSEC), 18th Asia Pacific, 2011, pp. 357-364.

[13] ECMA-International, "ECMAScript Language Specification," website, http://www.ecma-international.org/.

[14] F. Yang, J.-P. Jacquot, and J. Souquières, "The case for Using Simulation to Validate Event-B Specifications," in Software Engineering Conference (APSEC), 19th Asia-Pacific, vol. 1, 2012, pp. 85-90.

[15] D. Bert, M.-L. Potet, and N. Stouls, "Genesyst: a tool to reason about behavioral aspects of $B$ event specifications. application to security properties," in $Z B$ 2005: Formal Specification and Development in $Z$ and B, 4th International Conference of $B$ and $Z$ Users, ser. LNCS, vol. 3455. Springer-Verlag, 2005, pp. 299-318.

[16] F. Yang, "A Simulation Framework for the Validation of Event-B Specifications," Ph.D. dissertation, Université de Lorraine, 2013.

[17] J. T. Schwartz, R. B. Dewar, E. Schonberg, and E. Dubinsky, Programming with sets; an introduction to SETL. Springer-Verlag, 1986.

[18] A. Mashkoor and J.-P. Jacquot, "Stepwise Validation of Formal Specifications," in The eighteenth Asia-Pacific Software Engineering Conference (APSEC 2011). Ho Chi Minh City, Vietnam,2011 